\title{
Arithmetical properties of real numbers related to beta-expansions *
}

\author{
Kaneko Hajime ${ }^{\dagger}$
}

\begin{abstract}
The main purpose of this paper is to study the arithmetical properties of values $\sum_{m=0}^{\infty} \beta^{-w(m)}$, where $\beta$ is a fixed Pisot or Salem number and $w(m)(m=0,1, \ldots)$ are distinct sequences of nonnegative integers with $w(m+1)>w(m)$ for any sufficiently large $m$. We first introduce criteria for the algebraic independence of such values. Our criteria are applicable to certain sequences $w(m)(m=0,1, \ldots)$ with $\lim _{m \rightarrow \infty} w(m+1) / w(m)=$ 1. For example, we prove that two numbers

$$
\sum_{m=1}^{\infty} \beta^{-\lfloor\varphi(1,0 ; m)\rfloor}, \sum_{m=3}^{\infty} \beta^{-\lfloor\varphi(0,1 ; m)\rfloor}
$$

are algebraically independent, where $\varphi(1,0 ; m)=m^{\log m}$ and $\varphi(0,1 ; m)=$ $m^{\log \log m}$

Moreover, we also give criteria for linear independence of real numbers. Our criteria are applicable to the values $\sum_{m=0}^{\infty} \beta^{-\left\lfloor m^{\rho}\right\rfloor}$, where $\beta$ is a Pisot or Salem number and $\rho$ is a real number greater than 1 .
\end{abstract}

\section{Introduction}

Throughout this paper, we denote the set of nonnegative integers (resp. positive integers) by $\mathbb{N}$ (resp. $\mathbb{Z}^{+}$). We write the integral and fractional parts of a real number $x$ by $\lfloor x\rfloor$ and $\{x\}$, respectively. Moreover, $\lceil x\rceil$ is the minimal integer not less than $x$. We use the Vinogradov symbols $\gg$ and $\ll$, as well as the Landau symbols $O, o$ with their regular meanings. Finally, $f \sim g$ means that the ratio $f / g$ tends to 1

In what follows, we investigate the arithmetical properties of the values of power series $f(X)$ at algebraic points. For simplicity, we first consider the case where $f(X)$ has the form

$$
f(X)=\sum_{m=0}^{\infty} X^{w(m)}
$$

where $(w(m))_{m=0}^{\infty}$ is a sequence of nonnegative integers satisfying $w(m)<$ $w(m+1)$ for any sufficiently large $m$. We call $f(X)$ a gap series if

$$
\lim _{m \rightarrow \infty} \frac{w(m+1)}{w(m)}=\infty .
$$

\footnotetext{
*2010 Mathematics Subject Classification : primary 11J91; secondary 11K16, 11J72

$\dagger$ Keywords and phrases: algebraic independence, power series, beta expansion, Pisot numbers, Salem numbers.
} 
We say that $f(X)$ is a lacunary series if

$$
\liminf _{m \rightarrow \infty} \frac{w(m+1)}{w(m)}>1 .
$$

Note that if $f(X)$ is a lacunary series, then there exists a positive real number $\delta$ such that

$$
w(m)>(1+\delta)^{m}
$$

for any sufficiently large $m$.

In the rest of this secction, suppose that $\alpha$ is an algebraic number with $0<|\alpha|<1$. In paper [7, Bugeaud posed a problem on the transcendence of the values of power series $f(X)$ as follows: If $(w(m))_{m=0}^{\infty}$ increases sufficiently rapidly, then $\sum_{m=0}^{\infty} \alpha^{w(m)}$ is transcendental.

Corvaja and Zannier [8] showed that if $f(X)=\sum_{m=0}^{\infty} X^{w(m)}$ is a lacunary series, then $\sum_{m=0}^{\infty} \alpha^{w(m)}$ is transcendental. For instance, let $x, y$ be real numbers with $x>0$ and $y>1$. Then two numbers

$$
\sum_{m=0}^{\infty} \alpha^{\lfloor x(m !)\rfloor}, \sum_{m=0}^{\infty} \alpha^{\left\lfloor y^{m}\right\rfloor}
$$

are transcendental.

Adamczewski [1 improved the result above in the case of $\alpha=\beta^{-1}$, where $\beta$ is a Pisot or Salem number. Recall that Pisot numbers are algebraic integers greater than 1 whose conjugates except themselves have absolute values less than 1. Note that any rational integers greater than 1 are Pisot numbers. Salem numbers are algebraic integers greater than 1 such that the conjugates except themselves have moduli less than 1 and that there exists at least one conjugate with modulus 1 . Adamczewski 1 showed that if

$$
\liminf _{m \rightarrow \infty} \frac{w(m+1)}{w(m)}>1,
$$

then $\sum_{m=0}^{\infty} \beta^{-w(m)}$ is transcendental for any Pisot or Salem number $\beta$.

We now introduce known results on the algebraic independence of certain lacunary series at fixed algebraic points. First we consider the case where $f(X)$ is a gap series. Durand [10] showed that if $\alpha$ is a real algebraic number with $0<\alpha<1$, then the continuum set

$$
\left\{\sum_{m=0}^{\infty} \alpha^{\lfloor x(m !)\rfloor} \mid x \in \mathbb{R}, x>0\right\}
$$

is algebraically independent. Moreover, Shiokawa [17] gave a criterion for the algebraic independence of the values of certain gap series. Using his criterion, we deduce for general algebraic number $\alpha$ with $0<|\alpha|<1$ that the set (1.1) is algebraically independent.

Next, we consider the case where $f(X)$ is not a gap series. Using Mahler's method for algebraic independence, Nishioka [15] proved that the set

$$
\left\{\sum_{m=0}^{\infty} \alpha^{k^{m}} \mid k=2,3, \ldots\right\}
$$


is algebraically independent. Moreover, Tanaka 18 showed that if positive real numbers $w_{1}, \ldots, w_{m}$ are linearly independent over $\mathbb{Q}$, then the set

$$
\left\{\sum_{m=0}^{\infty} \alpha^{\left\lfloor w_{i} k^{m}\right\rfloor} \mid i=1, \ldots, m, k=2,3, \ldots\right\}
$$

is algebraically independent.

On the other hand, it is generally difficult to study algebraic independence in the case where $f(X)$ is not lacunary. In Section 2 we review known results on the criteria for transcendence of the value $\sum_{m=0}^{\infty} \beta^{-w(m)}$, where $\beta$ is a Pisot or Salem number and $(w(m))_{m=0}^{\infty}$ is a certain sequence of nonnegative integers with

$$
\lim _{m \rightarrow \infty} \frac{w(m+1)}{w(m)}=1 .
$$

In Section 3 we give the main results on the algebraic independence of real numbers applicable to

$$
\sum_{m=1}^{\infty} \beta^{-\left\lfloor m^{\log m}\right\rfloor}, \sum_{m=3}^{\infty} \beta^{-\left\lfloor m^{\log \log m}\right\rfloor} .
$$

In the same section we also investigate the linear independence of real numbers applicable to $\sum_{m=0}^{\infty} \beta^{-\left\lfloor m^{\rho}\right\rfloor}$ for a real number $\rho>1$. The main criteria for algebraic independence and linear independence, which are used to prove the main results, are denoted in Section 4. For the proof of the algebraic independence and linear independence, we need no functional equation because our criteria are flexible. We prove the main results in Section 5. Moreover, we show the criteria in Section 6.

\section{Transcendental results related to the numbers of nonzero digits}

In this section we review criteria for the transcendence of the value $\sum_{n=0}^{\infty} t_{n} \beta^{-n}$, where $\left(t_{n}\right)_{n=0}^{\infty}$ is a bounded sequence of nonnegative integers and $\beta$ is a Pisot or Salem number. First we consider the case where $\beta=b$ is an integer greater than 1 . We denote the base- $b$ expansion of a real number $\eta$ by

$$
\eta=\sum_{n=0}^{\infty} s_{n}^{(b)}(\eta) b^{-n},
$$

where $s_{0}^{(b)}(\eta)=\lfloor\eta\rfloor$ and $s_{n}^{(b)}(\eta) \in\{0,1, \ldots, b-1\}$ for any positive integer $n$. We may assume that $s_{n}^{(b)}(\eta) \leq b-2$ for infinitely many $n$ 's. For any positive integer $N$, put

$$
\lambda_{b}(\eta ; N):=\operatorname{Card}\left\{n \in \mathbb{N} \mid n<N, s_{n}^{(b)}(\eta) \neq 0\right\},
$$

where Card denotes the cardinality. 
Borel [5] conjectured for each integral base $b \geq 2$ that any algebraic irrational number is normal in base- $b$, which is still an open problem. For any real number $\rho>1$, put

$$
\gamma(\rho ; X):=\sum_{m=0}^{\infty} X^{\left\lfloor m^{\rho}\right\rfloor} .
$$

If Borel's conjecture is true, then $\gamma\left(\rho ; b^{-1}\right)$ is transcendental because $\gamma\left(\rho ; b^{-1}\right)$ is a non-normal irrational number in base- $b$. However, the transcendence of such values is not known except the case of $\rho=2$. If $\rho=2$, then Duverney, Nishioka, Nishioka, Shiokawa 11 and Bertrand 4 independently proved for any algebraic number $\alpha$ with $0<|\alpha|<1$ that $\gamma(2 ; \alpha)$ is transcendental.

Bailey, Borwein, Crandall, and Pomerance [3. gave a criterion for the transcendence of real numbers, using lower bounds for the numbers of nonzero digits in the binary expansions of algebraic irrational numbers. Let $\eta$ be an algebraic irrational number with degree $D$. Bailey, Borwein, Crandall, and Pomerance [3] showed that there exist positive constants $C_{1}(\eta)$ and $C_{2}(\eta)$, depending only on $\eta$, satisfying

$$
\lambda_{2}(\eta ; N) \geq C_{1}(\eta) N^{1 / D}
$$

for any integer $N$ with $N \geq C_{2}(\eta)$. Note that $C_{1}(\eta)$ is effectively computable but $C_{2}(\eta)$ is not. For any integral base $b \geq 2$, Adamczewski, Faverjon [2] and Bugeaud [6] gave effective versions of lower bounds for $\lambda_{b}(\eta ; N)$ as follows: There exist effectively computable positive constants $C_{3}(b, \eta)$ and $C_{4}(b, \eta)$, depending only on $b$ and $\eta$, satisfying

$$
\lambda_{b}(\eta ; N) \geq C_{3}(b, \eta) N^{1 / D}
$$

for any integer $N$ with $N \geq C_{4}(b, \eta)$. Using (2.1), we obtain for any real number $\rho>1$ that $\gamma\left(\rho ; b^{-1}\right)$ is not an algebraic number of degree less than $\rho$. In fact, $\gamma\left(\rho ; b^{-1}\right)$ is an irrational number satisfying

$$
\lambda_{b}\left(\gamma\left(\rho ; b^{-1}\right) ; N\right) \sim N^{1 / \rho}
$$

as $N$ tends to infinity. Thus, (2.1) does not hold if $D<\rho$.

By (2.1), we also deduce a criterion for the transcendence of real numbers as follows: Let $\eta$ be a positive irrational number. Suppose for any real positive real number $\varepsilon$ that

$$
\liminf _{N \rightarrow \infty} \frac{\lambda_{b}(\eta ; N)}{N^{\varepsilon}}=0
$$

Then $\eta$ is a transcendental number. Note that the criterion above was essentially obtained by Bailey, Borwein, Crandall, and Pomerance [3]. Note that if $\sum_{m=0}^{\infty} X^{w(m)}$ is lacunary, then $\eta=\sum_{m=0}^{\infty} b^{-w(m)}$ satisfies (2.2) by

$$
\lambda_{b}(\eta ; N)=O(\log N) .
$$

We give another example of transcendental numbers. For any real numbers $y>0$ and $R \geq 1$, we put

$$
\varphi(y ; R):=\exp \left((\log R)^{1+y}\right)=R^{(\log R)^{y}} .
$$


Moreover, we set

$$
\xi(y ; X):=1+\sum_{m=1}^{\infty} X^{\lfloor\varphi(y ; m)\rfloor} .
$$

Note that $\xi(y ; X)$ is not lacunary by

$$
\lim _{m \rightarrow \infty} \frac{\varphi(y ; m+1)}{\varphi(y ; m)}=1 .
$$

We get that $\eta:=\xi\left(y ; b^{-1}\right)$ is transcendental for any integer $b \geq 2$ because $\eta$ satisfies (2.2).

In what follows, we consider the case where $\beta$ is a general Pisot or Salem number. We introduce results in 14 related to the $\beta$-expansion of algebraic numbers. For any formal power series $f(X)=\sum_{n=0}^{\infty} t_{n} X^{n}$, we put

$$
S(f):=\left\{n \in \mathbb{N} \mid t_{n} \neq 0\right\} .
$$

Moreover, for any nonempty set $\mathcal{A}$ of nonnegative integers, we set

$$
\lambda(\mathcal{A} ; N):=\operatorname{Card}(\mathcal{A} \cap[0, N)) .
$$

We denote the degree of a field extension $L / K$ by $[L: K]$.

THEOREM 2.1 ([14]). Let $A$ be a positive integer and let $f(X)=\sum_{n=0}^{\infty} t_{n} X^{n}$ be a power series with integral coefficients. Assume that $0 \leq t_{n} \leq A$ for any nonnegative integer $n$ and that there exist infinitely many $n$ 's satisfying $t_{n} \neq 0$. Let $\beta$ be a Pisot or Salem number. Suppose that $\eta=f\left(\beta^{-1}\right)$ is an algebraic number with $[\mathbb{Q}(\beta, \eta): \mathbb{Q}(\beta)]=D$. Then there exist effectively computable positive constants $C_{5}(A, \beta, \eta)$ and $C_{6}(A, \beta, \eta)$, depending only on $A, \beta$ and $\eta$ satisfying

$$
\lambda(S(f) ; N) \geq C_{5}(A, \beta, \eta)\left(\frac{N}{\log N}\right)^{1 / D}
$$

for any integer $N$ with $N \geq C_{6}(A, \beta, \eta)$.

In the rest of this section, let $\beta$ be a Pisot or Salem number. Using Theorem 2.1 we obtain for any real number $\rho>1$ that

$$
\left[\mathbb{Q}\left(\gamma\left(\rho ; \beta^{-1}\right), \beta\right): \mathbb{Q}(\beta)\right] \geq\lceil\rho\rceil
$$

by

$$
\lambda(S(\gamma(\rho ; X)) ; N) \sim N^{1 / \rho}
$$

as $N$ tends to infinity.

Note that Theorem 2.1 is applicable to the study of the nonzero digits in the $\beta$-expansions of algebraic numbers. We recall the definition of $\beta$-expansion defined by Rényi [16] in 1957. Let $T_{\beta}:[0,1) \rightarrow[0,1)$ be the $\beta$-transformation defined by $T_{\beta}(x)=\{\beta x\}$ for $x \in[0,1)$. Then the $\beta$-expansion of a real number $\eta \in[0,1)$ is denoted as

$$
\eta=\sum_{n=1}^{\infty} s_{n}^{(\beta)}(\eta) \beta^{-n}
$$


where $s_{n}^{(\beta)}(\eta)=\left\lfloor\beta T_{\beta}^{n-1}(\eta)\right\rfloor$ for any $n \geq 1$. Note that $0 \leq s_{n}^{(\beta)}(\eta) \leq\lfloor\beta\rfloor$ for any $n \geq 1$. Put

$$
\lambda_{\beta}(\eta ; N):=\operatorname{Card}\left\{n \in \mathbb{Z}^{+}, n \leq N, s_{n}^{(\beta)}(\eta) \neq 0\right\}
$$

for any positive integer $N$. Applying Theorem 2.1 with $B=\lfloor\beta\rfloor$, we deduce that if $\eta \in[0,1)$ is an algebraic number with $[\mathbb{Q}(\beta, \eta): \mathbb{Q}(\beta)]=D$, then

$$
\lambda_{\beta}(\eta ; N) \gg\left(\frac{N}{\log N}\right)^{1 / D}
$$

for any sufficiently large integer $N$.

Using Theorem 2.1 we also deduce a criterion for the transcendence of real numbers as follows: Let $f(X)$ be a power series whose coefficients are bounded nonnegative integers. Suppose that $f(X)$ is not a polynomial and that

$$
\liminf _{m \rightarrow \infty} \frac{\lambda_{\beta}(S(f) ; N)}{N^{\varepsilon}}=0
$$

for any positive real number $\varepsilon$. Then $f\left(\beta^{-1}\right)$ is transcendental. Note that the criterion above was already obtained in 13 and that the criterion is applicable even if the representation $\sum_{n=0}^{\infty} t_{n} \beta^{-n}$ does not coincide with the $\beta$-expansion of $f\left(\beta^{-1}\right)$. In the same way as the case where $\beta=b \geq 2$ is an integer, we obtain for any positive real number $y$ that $\xi\left(y ; \beta^{-1}\right)$ is transcendental.

In the end of this section we introduce a corollary of Theorem 2.1, which we need to prove our criteria for linear independence.

COROLLARY 2.2. Let $A$ be a positive integer and $f(X)$ a nonpolynomial power series whose coefficients are bounded nonnegative integers. Assume that there exists a positive real number $\delta$ satisfying

$$
\lambda(S(f) ; R)<R^{-\delta+1 / A}
$$

for infinitely many integer $R \geq 0$. Then, for any Pisot or Salem number $\beta$, we have

$$
\left[\mathbb{Q}\left(f\left(\beta^{-1}\right), \beta\right): \mathbb{Q}(\beta)\right] \geq A+1 .
$$

\section{Main results}

\subsection{Results on algebraic independence}

We use the same notation as Section 2.

THEOREM 3.1. Let $\beta$ be a Pisot or Salem number. Then the continuum set

$$
\left\{\xi\left(y ; \beta^{-1}\right) \mid y \in \mathbb{R}, y \geq 1\right\}
$$

is algebraically independent.

Note that if $\beta=b$ is an integer greater than 1 , then the algebraic independence of (3.1) was proved in 12. However, the algebraic independence of the set

$$
\left\{\xi\left(y ; b^{-1}\right) \mid y \in \mathbb{R}, y>0\right\}
$$


is unknown.

On the other hand, considering the algebraic independence of two values, we obtain more detailed results. Set

$$
\Theta:=\left\{(y, z) \in \mathbb{R}^{2} \mid y>0 \text {, or } y=0 \text { and } z>0\right\} .
$$

Moreover, for any real number $R \geq 3$ and $(y, z) \in \Theta$, we put

$$
\begin{aligned}
\varphi(y, z ; R) & :=\exp \left((\log R)^{1+y}(\log \log R)^{z}\right) \\
& =R^{(\log R)^{y}(\log \log R)^{z}}
\end{aligned}
$$

and

$$
\xi(y, z ; X):=1+\sum_{m=3}^{\infty} X^{\lfloor\varphi(y, z ; m)\rfloor} .
$$

THEOREM 3.2. Let $\left(y_{1}, z_{1}\right)$ and $\left(y_{2}, z_{2}\right)$ be distinct elements in $\Theta$. Then the two values $\xi\left(y_{1}, z_{1} ; \beta^{-1}\right)$ and $\xi\left(y_{2}, z_{2} ; \beta^{-1}\right)$ are algebraically independent for any Pisot or Salem number $\beta$.

Considering the case of $z_{1}=z_{2}=0$ in Theorem 3.2, we get the following:

COROLLARY 3.3. Let $y_{1}$ and $y_{2}$ be distinct positive real numbers. Then the two values $\xi\left(y_{1} ; \beta^{-1}\right)$ and $\xi\left(y_{2} ; \beta^{-1}\right)$ are algebraically independent for any Pisot or Salem number $\beta$.

In the case where $\beta=b$ is an integer greater than 1 , the algebraic independence of the two values $\xi\left(y_{1} ; b^{-1}\right)$ and $\xi\left(y_{2} ; b^{-1}\right)$ was obtained in [12].

Applying Theorem 3.2 with $\left(y_{1}, z_{1}\right)=(1,0)$ and $\left(y_{2}, z_{2}\right)=(0,1)$, we deduce the following:

COROLLARY 3.4. For any Pisot or Salem number $\beta$ the two values

$$
\sum_{m=1}^{\infty} \beta^{-\left\lfloor m^{\log m}\right\rfloor}, \sum_{m=3}^{\infty} \beta^{-\left\lfloor m^{\log \log m}\right\rfloor}
$$

are algebraically independent.

In the last of this subsection, we introduce the algebraic independence of the values of $\xi(y, z ; X)$ and lacunary series.

THEOREM 3.5. Let $(y, z) \in \Xi$ and let $x$ be a real number greater than 1 . Then, $\xi\left(y, z, \beta^{-1}\right)$ and $\sum_{m=0}^{\infty} \beta^{-\left\lfloor x^{m}\right\rfloor}$ are algebraically independent for any Pisot or Salem number $\beta$.

\subsection{Results on linear independence}

Let $\mathcal{F}$ be the set of nonpolynomial power series $g(X)$ satisfying the following three assumptions:

1. The coefficients of $g(X)$ are bounded nonnegative integers. 
2. For an arbitrary positive real number $\varepsilon$, we have

$$
\lambda(S(g) ; R)=o\left(R^{\varepsilon}\right)
$$

as $R$ tends to infinity.

3. There exists a positive constant $C$ such that

$$
[R, C R] \cap S(g) \neq \emptyset
$$

for any sufficiently large $R$.

In order to state our results, we give a lemma on the zeros of certain polynomials. For any positive integer $k$, put

$$
G_{k}(X):=(1-X)^{k}+(k-1) X-1 .
$$

LEMMA 3.6. Suppose that $k \geq 3$. Then the following holds:

1) There exists a unique zero $\sigma_{k}$ of $G_{k}(X)$ on the interval $(0,1)$.

2) Let $x$ be a real number with $0<x<1$. Then $G_{k}(x)<0\left(\right.$ resp. $\left.G_{k}(x)>0\right)$

if and only if $x<\sigma_{k}$ (resp. $\left.x>\sigma_{k}\right)$.

3) $\left(\sigma_{k}\right)_{k=3}^{\infty}$ is strictly decreasing.

Proof. Observe that $G_{k}^{\prime}(X)=-k(1-X)^{k-1}+k-1$ is monotone increasing on the interval $(0,1)$ and that $G_{k}^{\prime}(X)$ has a unique zero $\widetilde{\sigma_{k}}$ on $(0,1)$. Thus, $G_{k}(X)$ is monotonically decreasing on $\left(0, \widetilde{\sigma_{k}}\right]$ and monotonically increasing on $\left(\widetilde{\sigma_{k}}, 1\right)$. Hence, the first and second statements of the lemma follow from $G_{k}(0)=0$ and $G_{k}(1)=k-2>0$.

Next, we assume that $k \geq 4$. Using

$$
G_{k-1}\left(\sigma_{k-1}\right)=\left(1-\sigma_{k-1}\right)^{k-1}+(k-2) \sigma_{k-1}-1=0,
$$

we get

$$
G_{k}\left(\sigma_{k-1}\right)=\left(1-\sigma_{k-1}\right)^{k}+(k-1) \sigma_{k-1}-1=(k-2) \sigma_{k-1}^{2}>0 .
$$

Hence, we obtain $\sigma_{k}<\sigma_{k-1}$ by the second statement of the lemma.

THEOREM 3.7. Let $A$ be a positive integer and $\rho$ a real number. Suppose that

$$
\left\{\begin{array}{cl}
\rho>A & \text { if } A \leq 3 \\
\rho>\sigma_{A}^{-1} & \text { if } A \geq 4 .
\end{array}\right.
$$

Then, for any $g(X) \in \mathcal{F}$ and any Pisot or Salem number $\beta$, the set

$$
\left\{\gamma\left(\rho ; \beta^{-1}\right)^{k_{1}} g\left(\beta^{-1}\right)^{k_{2}} \mid k_{1}, k_{2} \in \mathbb{N}, k_{1} \leq A\right\}
$$

is linearly independent over $\mathbb{Q}(\beta)$.

We give numerical examples of $\sigma_{n}^{-1}(n \geq 4)$ as follows:

$$
\sigma_{4}^{-1}=5.278 \ldots, \sigma_{5}^{-1}=8.942 \ldots, \sigma_{6}^{-1}=13.60 \ldots
$$


COROLLARY 3.8. Let $A, \rho$ be as in Theorem 3.7

1) For any real number $y>1$ and any Pisot or Salem number $\beta$, the set

$$
\left\{\gamma\left(\rho ; \beta^{-1}\right)^{k_{1}}\left(\sum_{m=0}^{\infty} \beta^{-\left\lfloor y^{m}\right\rfloor}\right)^{k_{2}} \mid k_{1}, k_{2} \in \mathbb{N}, k_{1} \leq A\right\}
$$

is linearly independent over $\mathbb{Q}(\beta)$.

2) For any $(y, z) \in \Theta$ and any Pisot or Salem number $\beta$, the set

$$
\left\{\gamma\left(\rho ; \beta^{-1}\right)^{k_{1}} \xi\left(y, z ; \beta^{-1}\right)^{k_{2}} \mid k_{1}, k_{2} \in \mathbb{N}, k_{1} \leq A\right\}
$$

is linearly independent over $\mathbb{Q}(\beta)$.

Using the asymptotic behavior of the sequence $\left(\sigma_{m}\right)_{m=3}^{\infty}$, we deduce the following:

COROLLARY 3.9. Let $\varepsilon$ be an arbitrary positive real number. Then there exists an effectively computable positive constant $A_{0}(\varepsilon)$, depending only on $\varepsilon$ satisfying the following: Let $A$ be an integer with $A \geq A_{0}(\varepsilon)$ and $\rho$ a real number with $\rho>(\varepsilon+1 / 2) A^{2}$. Then, for any $g(X) \in \mathcal{F}$ and any Pisot or Salem number $\beta$, the set

$$
\left\{\gamma\left(\rho ; \beta^{-1}\right)^{k_{1}} g\left(\beta^{-1}\right)^{k_{2}} \mid k_{1}, k_{2} \in \mathbb{N}, k_{1} \leq A\right\}
$$

is linearly independent over $\mathbb{Q}(\beta)$.

\section{Criteria for algebraic independence and linear independence}

Let $k$ be a nonnegative integer and $f(X) \in \mathbb{Z}[[X]] \backslash \mathbb{Z}[X]$. We denote the Minkowski sum of $S(f)$ by

$$
k S(f):=\left\{\begin{array}{cc}
\{0\} & (k=0), \\
\left\{s_{1}+\cdots+s_{k} \mid s_{1}, \ldots, s_{k} \in S(f)\right\} & (k \geq 1) .
\end{array}\right.
$$

Moreover, for any $\left(k_{1}, \ldots, k_{r}\right) \in \mathbb{N}^{r}$ and $f_{1}(X), \ldots, f_{r}(X) \in \mathbb{Z}[[X]] \backslash \mathbb{Z}[X]$, we set

$$
\sum_{h=1}^{r} k_{h} S\left(f_{h}\right):=\left\{s_{1}+\cdots+s_{r} \mid s_{h} \in k_{h} S\left(f_{h}\right) \text { for } h=1, \ldots, r\right\} .
$$

REMARK 1. Suppose that $0 \in S\left(f_{i}\right)$ for $i=1, \ldots, r$. Then, for any $\left(k_{1}, \ldots, k_{r}\right) \in$ $\mathbb{N}^{r}$ and $\left(k_{1}^{\prime}, \ldots, k_{r}^{\prime}\right) \in \mathbb{N}^{r}$ with $k_{i} \geq k_{i}^{\prime}$ for any $i=1, \ldots, r$, we have

$$
\sum_{h=1}^{r} k_{h} S\left(f_{h}\right) \supset \sum_{h=1}^{r} k_{h}^{\prime} S\left(f_{h}\right) .
$$

Let $\mathcal{A}$ be a nonempty set of nonnegative integers and $R$ a real number with $R>\min \mathcal{A}$. Then we put

$$
\theta(R ; \mathcal{A}):=\max \{n \in \mathcal{A} \mid n<R\} .
$$


THEOREM 4.1. Let $A, r$ be integers with $A \geq 1$ and $r \geq 2$. Let $f_{i}(X)=$ $\sum_{n=0}^{\infty} t_{i}(n) X^{n}(i=1, \ldots, r)$ be nonpolynomial power series with integral coefficients. We assume that $f_{1}(X), \ldots, f_{r}(X)$ satisfy the following four assumptions:

1. There exists a positive constant $C_{7}$ satisfying

$$
0 \leq t_{i}(n) \leq C_{7}
$$

for any $i=1, \ldots, r$ and nonnegative integer $n$.

2. Let $k_{1}, \ldots, k_{r}$ be nonnegative integers. Suppose that

$$
\left\{\begin{array}{cl}
k_{1} \leq A-1 & \text { if } r=2 \\
k_{1} \leq A & \text { if } r \geq 3
\end{array}\right.
$$

Then

$$
\begin{aligned}
R-\theta\left(R ; \sum_{h=1}^{r-2} k_{h} S\left(f_{h}\right)\right. & \left.+\left(1+k_{r-1}\right) S\left(f_{r-1}\right)\right) \\
& =o\left(\frac{R}{\prod_{h=1}^{r} \lambda\left(S\left(f_{h}\right) ; R\right)^{k_{h}}}\right)
\end{aligned}
$$

as $R$ tends to infinity.

3. There exists a positive real number $\delta$ satisfying

$$
\lambda\left(S\left(f_{1}\right) ; R\right)=o\left(R^{-\delta+1 / A}\right)
$$

as $R$ tends to infinity. Moreover, for any $i=2, \ldots, r$ and any real number $\varepsilon$, we have

$$
\lambda\left(S\left(f_{i}\right) ; R\right)=o\left(\lambda\left(S\left(f_{i-1}\right) ; R\right)^{\varepsilon}\right)
$$

as $R$ tends to infinity.

4. There exist positive constants $C_{8}, C_{9}$ such that

$$
\left[R, C_{8} R\right] \cap S\left(f_{r}\right) \neq \emptyset
$$

for any real number $R$ with $R \geq C_{9}$.

Then, for any Pisot or Salem number $\beta$, the set

$$
\left\{f_{1}\left(\beta^{-1}\right)^{k_{1}} f_{2}\left(\beta^{-1}\right)^{k_{2}} \cdots f_{r}\left(\beta^{-1}\right)^{k_{r}} \mid k_{1}, k_{2}, \ldots, k_{r} \in \mathbb{N}, k_{1} \leq A\right\}
$$

is linearly independent over $\mathbb{Q}(\beta)$.

Let $a(R)$ be a real valued function defined on an interval $\left[R_{0}, \infty\right)$ with $R_{0} \in$ $\mathbb{R}$. We say that $a(R)$ ultimately increasing if $a(R)$ is strictly increasing for any sufficiently large real number $R$. Similarly, we say that $(a(m))_{m=m_{0}}^{\infty}$ is ultimately increasing if this sequence is strictly increasing for any sufficiently large integer $m$. 
THEOREM 4.2. Let $a(R), u(R)$ be ultimately increasing functions defined on $\left[m_{0}, \infty\right)$ with $m_{0} \in \mathbb{N}$. Assume that $(\lfloor a(m)\rfloor)_{m=m_{0}}^{\infty}$ and $(\lfloor u(m)\rfloor)_{m=m_{0}}^{\infty}$ are also ultimately increasing. Let $b(R), v(R)$ be the inverse functions of $a(R), u(R)$, respectively, for any sufficiently large $R$. Assume that a $(R)$ satisfies the following two assumptions:

1. $(\log a(R)) /(\log R)$ is ultimately increasing and

$$
\lim _{R \rightarrow \infty} \frac{\log a(R)}{\log R}=\infty
$$

2. We have $a(R)$ is differentiable. Moreover, for an arbitrary positive real number $\varepsilon$, there exists a positive constant $C_{10}(\varepsilon)$, depending only on $\varepsilon$, such that

$$
(\log a(R))^{\prime}<R^{-1+\varepsilon}
$$

for any real number $R$ with $R \geq C_{10}(\varepsilon)$.

Moreover, suppose that $u(R)$ fulfills the following two assumptions:

1. There exists a positive constant $C_{11}$ such that

$$
\frac{u(R+1)}{u(R)}<C_{11}
$$

for any sufficiently large real number $R$.

2.

$$
\lim _{R \rightarrow \infty} \frac{\log b(R)}{\log v(R)}=\infty
$$

Then, for any Pisot or Salem number $\beta$, the two numbers

$$
\sum_{m=m_{0}}^{\infty} \beta^{-\lfloor a(m)\rfloor}, \sum_{m=m_{0}}^{\infty} \beta^{-\lfloor u(m)\rfloor}
$$

are algebraically independent.

\section{Proof of main results}

In this section we prove results in Section 3, using Theorems 4.1 and 4.2 .

\subsection{Proof of results on algebraic independence}

Proof of Theorem 3.1. Let $y_{1}, y_{2}, \ldots, y_{r}$ be real numbers with $1 \leq y_{1}<y_{2}<$ $\cdots<y_{r}$. We show that $f_{i}(X):=\xi\left(y_{i} ; X\right)(i=1, \ldots, r)$ fulfill the assumptions in Theorem 4.1 for any positive integer $A$. The first assumption is clear. Recall that we proved Theorem 1.3 in [12, showing for any integer $b \geq 2$ that $f_{1}\left(b^{-1}\right), \ldots, f_{r}\left(b^{-1}\right)$ satisfy the assumptions of Theorem 2.1 in [12. In the same way, we can check that $f_{1}(X), \ldots, f(X)$ fulfill the third and fourth assumptions in Theorem 4.1 . 
In what follows, we verify the second assumption. Let $y$ be a fixed positive real number. Then we denote the inverse function of $\varphi(y ; R)$ by

$$
\psi(y ; R)=\exp \left((\log R)^{1 /(1+y)}\right) .
$$

For $i=1, \ldots, r$, we have

$$
\lambda\left(S\left(f_{i}\right) ; R\right) \sim \psi\left(y_{i} ; R\right)
$$

as $R$ tends to infinity.

LEMMA 5.1. Let $\boldsymbol{k}=\left(k_{1}, \ldots, k_{r}\right) \in \mathbb{N}^{r} \backslash\{(0, \ldots, 0)\}$. Then

$$
R-\theta\left(R ; \sum_{i=1}^{r} k_{i} S\left(f_{i}\right)\right) \ll \frac{R(\log R)^{k_{1}+\cdots+k_{r}}}{\prod_{i=1}^{r} \psi\left(y_{i} ; R\right)^{k_{i}}}
$$

for any real $R$ with $R \geq 2$.

Proof. We can show Lemma 5.1 in the same way as the proof of Lemma 3.1 in [12.

Let $A$ be any positive integer and $k_{1}, \ldots, k_{r}$ any nonnegative integers. Without loss of generality, we may assume that $k_{r} \geq 1$. Applying Lemma 5.1 with $\boldsymbol{k}=\left(k_{1}, \ldots, k_{r-2}, 1+k_{r-1}, 0\right) \in \mathbb{N}^{r} \backslash\{(0, \ldots, 0)\}$, we get for any $R \geq 2$ that

$$
\begin{aligned}
R-\theta\left(R ; \sum_{h=1}^{r-2} k_{h} S\left(f_{h}\right)\right. & \left.+\left(1+k_{r-1}\right) S\left(f_{r-1}\right)\right) \\
= & o\left(\frac{R(\log R)^{1+k_{1}+\cdots+k_{r-1}}}{\psi\left(y_{r-1} ; R\right) \prod_{h=1}^{r-1} \psi\left(y_{i} ; R\right)^{k_{i}}}\right) .
\end{aligned}
$$

Observe that

$$
\begin{aligned}
\log \left((\log R)^{1+k_{1}+\cdots+k_{r-1}}\right) & \ll \log \log R \\
& =o\left((\log R)^{1 /\left(1+y_{r-1}\right)}\right) \\
& =o\left(\frac{1}{2} \log \psi\left(y_{r-1} ; R\right)\right)
\end{aligned}
$$

as $R$ tends to infinity. Thus, we see

$$
(\log R)^{1+k_{1}+\cdots+k_{r-1}}=o\left(\psi\left(y_{r-1} ; R\right)^{1 / 2}\right) .
$$

Combining (5.1) and (5.2), we obtain

$$
\begin{aligned}
& R-\theta\left(R ; \sum_{h=1}^{r-2} k_{h} S\left(f_{h}\right)+\left(1+k_{r-1}\right) S\left(f_{r-1}\right)\right) \\
& =o\left(\frac{R}{\psi\left(y_{r-1} ; R\right)^{1 / 2} \prod_{h=1}^{r-1} \psi\left(y_{i} ; R\right)^{k_{i}}}\right)=o\left(\frac{R}{\prod_{h=1}^{r} \psi\left(y_{i} ; R\right)^{k_{i}}}\right),
\end{aligned}
$$

where we use the third assumption in Theorem 4.1 with $i=r$ and $\varepsilon=1 /\left(2 k_{r}\right)$ for the last equality. Therefore, we checked the second assumption. 
Proof of Theorem [3.2. Without loss of generality, we may assume that $y_{1}<y_{2}$, or $y_{1}=y_{2}$ and $z_{1}<z_{2}$. Put

$$
a(R):=\varphi\left(y_{1}, z_{1} ; R\right), u(R):=\varphi\left(y_{2}, z_{2} ; R\right) .
$$

In what follows, we check that $a(R), u(R)$ satisfy the assumptions in Theorem 4.2 Note that $a(R), u(R)(R \geq 3)$ and $(\lfloor a(m)\rfloor)_{m=3}^{\infty},(\lfloor u(m)\rfloor)_{m=3}^{\infty}$ are ultimately increasing. The assumptions on $a(R)$ in Theorem 4.2 are easily checked. In fact, the first assumption holds by

$$
\frac{\log a(R)}{\log R}=(\log R)^{y_{1}}(\log \log R)^{z_{1}} .
$$

Moreover, the second assumption follows from

$$
\begin{aligned}
& (\log a(R))^{\prime} \\
& =\left\{\begin{array}{cc}
\left(1+y_{1}\right)(\log R)^{y_{1}} / R & \text { if } z_{1}=0, \\
(\log R)^{y_{1}}(\log \log R)^{-1+z_{1}}\left(z_{1}+\left(1+y_{1}\right) \log \log R\right) / R & \text { if } z_{1} \neq 0 .
\end{array}\right.
\end{aligned}
$$

Calculating $(\log u(R))^{\prime}$ in the same way as (5.3), we see

$$
\lim _{R \rightarrow \infty}(\log u(R))^{\prime}=0 .
$$

Using the mean value theorem, we get

$$
\lim _{R \rightarrow \infty} \frac{u(R+1)}{u(R)}=1
$$

which implies the first assumption on $u(R)$ in Theorem 4.2 .

We now check the second assumption on $u(R)$. Using

$$
\log a(R)=(\log R)^{1+y_{1}}(\log \log R)^{z_{1}},
$$

we get

$$
\log R=(\log b(R))^{1+y_{1}}(\log \log b(R))^{z_{1}} .
$$

Similarly,

$$
\log R=(\log v(R))^{1+y_{2}}(\log \log v(R))^{z_{2}} .
$$

First we assume that $y_{1}<y_{2}$. Put $d:=y_{2}-y_{1}>0$. By (5.5) and (5.6), we get

$$
(\log v(R))^{1+y_{1}+(2 d) / 3}<\log R<(\log b(R))^{1+y_{1}+d / 3}
$$

for any sufficiently large $R$. Consequently, we obtain

$$
(\log v(R))^{d / 3}<\left(\frac{\log b(R)}{\log v(R)}\right)^{1+y_{1}+d / 3}
$$

which implies (4.4).

Next we assume that $y_{1}=y_{2}=: y$ and $z_{1}<z_{2}$. Using (5.5) and (5.6) again, we see

$$
\frac{(\log \log v(R))^{z_{2}}}{(\log \log b(R))^{z_{1}}}=\left(\frac{\log b(R)}{\log v(R)}\right)^{1+y}
$$


Taking the logarithm of the both-hand sides of (5.7), we get

$$
\begin{aligned}
& z_{2} \log \log \log v(R)-z_{1} \log \log \log b(R) \\
& =(1+y) \log \log b(R)-(1+y) \log \log v(R)
\end{aligned}
$$

Note that $b(R) \geq v(R)$ for any sufficiently large $R$. Thus, dividing (5.8) by $\log \log b(R)$, we see

$$
\lim _{R \rightarrow \infty} \frac{\log \log v(R)}{\log \log b(R)}=1 .
$$

Combining (5.7), (5.9), and $z_{2}>z_{1}$, we deduce (4.4).

Proof of Theorem 3.5. Applying Theorem 4.2 with

$$
a(R):=\varphi(y, z ; R), u(R):=x^{R},
$$

we deduce Theorem 3.5. In fact, we can check the assumptions on $a(R)$ in Theorem 4.2 in the same way as the proof of Theorem 3.2. Moreover, (4.4) is seen by (5.5) and $v(R)=(\log R) /(\log x)$.

\subsection{Proof of results on linear independence}

Proof of Theorem 3.7. We show that the assumptions on Theorem 4.1 are satisfied, where $A$ is defined as in Theorem 3.7 $r=2, f_{1}(X):=\gamma(\rho ; X)$, and $f_{2}(X):=g(X)$. The first assumption is clear. The fourth assumption follows from the third assumption on $\mathcal{F}$.

In order to check the third assumption, it suffices to show that

$$
\frac{1}{\rho}<\frac{1}{A}
$$

by (2.3) and the second assumption on $\mathcal{F}$. We may assume that $A \geq 4$ by (3.2). Using

$$
\begin{aligned}
\log \left(1-\frac{1}{A}\right)^{A} & =-A \sum_{n=1}^{\infty} \frac{1}{n} A^{-n} \\
& >-A \sum_{n=1}^{\infty} A^{-n}=-1-\frac{1}{A-1},
\end{aligned}
$$

we get by $A \geq 4$ that

$$
\begin{aligned}
\left(1-\frac{1}{A}\right)^{A} & >\exp \left(-1-\frac{1}{A-1}\right) \\
& \geq \exp \left(-\frac{4}{3}\right)>\frac{1}{4} \geq \frac{1}{A} .
\end{aligned}
$$

Hence, we obtain

$$
G_{A}\left(\frac{1}{A}\right)=\left(1-\frac{1}{A}\right)^{A}-\frac{1}{A}>0,
$$

which implies (5.10) by (3.2) and the second statement of Lemma 3.6. In what follows, we check the second assumption of Theorem 4.1. The following lemma was inspired by the results of Daniel 9 . 
LEMMA 5.2. Let $k$ be a positive integer. Then

$$
R-\theta\left(R ; k S\left(f_{1}\right)\right)=O\left(R^{(1-1 / \rho)^{k}}\right)
$$

for any $R \geq 1$, where the implied constant in the symbol $O$ does not depend on $R$, but on $k$.

Proof. First we consider the case of $k=1$. Using the mean value theorem, we see that

$$
\begin{aligned}
\left\lfloor(m+1)^{\rho}\right\rfloor-\left\lfloor m^{\rho}\right\rfloor & =(m+1)^{\rho}-m^{\rho}+O(1) \\
& =O\left(m^{\rho-1}\right)=O\left(\left\lfloor m^{\rho}\right\rfloor^{1-1 / \rho}\right)
\end{aligned}
$$

for any positive integer $m$. For any sufficiently large $R$, take a positive integer $m$ with

$$
\left\lfloor m^{\rho}\right\rfloor<R \leq\left\lfloor(m+1)^{\rho}\right\rfloor
$$

Then we get

$$
R-\theta\left(R ; S\left(f_{1}\right)\right) \leq\left\lfloor(m+1)^{\rho}\right\rfloor-\left\lfloor m^{\rho}\right\rfloor=O\left(R^{1-1 / \rho}\right)
$$

by (5.12).

Next, we assume that (5.11) holds for a positive integer $k$. Let

$$
R_{0}:=R-\theta\left(R ; k S\left(f_{1}\right)\right) \in \mathbb{Z}^{+} .
$$

The inductive hypothesis implies that

$$
R_{0}=O\left(R^{(1-1 / \rho)^{k}}\right) .
$$

Set

$$
\eta:=\theta\left(R ; k S\left(f_{1}\right)\right)+\theta\left(R_{0} ; S\left(f_{1}\right)\right) .
$$

Then we have $\eta \in(k+1) S\left(f_{1}\right)$ and

$$
R-\eta=R_{0}-\theta\left(R_{0} ; S\left(f_{1}\right)\right)>0 .
$$

Thus,

$$
\theta\left(R ;(k+1) S\left(f_{1}\right)\right) \geq \eta .
$$

Combining (5.14) and (5.15), we obtain

$$
\begin{aligned}
R-\theta\left(R ;(k+1) S\left(f_{1}\right)\right) & \leq R-\eta \\
& =R_{0}-\theta\left(R_{0} ; S\left(f_{1}\right)\right)
\end{aligned}
$$

Consequently, using (5.11) with $k=1$ and $R=R_{0}$, we deduce that

$$
\begin{aligned}
0 & <R-\theta\left(R ;(k+1) S\left(f_{1}\right)\right) \\
& =O\left(R_{0}^{1-1 / \rho}\right)=O\left(R^{(1-1 / \rho)^{k+1}}\right)
\end{aligned}
$$

by (5.13). 
Using Lemma 5.2 with $k=1+k_{1}$, we get

$$
\begin{aligned}
\log _{R} F_{1}(R) & :=\log _{R}\left(R-\theta\left(R ;\left(1+k_{1}\right) S\left(f_{1}\right)\right)\right) \\
& \leq\left(1-\frac{1}{\rho}\right)^{1+k_{1}}+o(1)
\end{aligned}
$$

as $R$ tends to infinity. Moreover, using (2.3) and the second assumption on $\mathcal{F}$, we see

$$
\log _{R} F_{2}(R):=\log _{R}\left(\frac{R}{\prod_{i=1}^{2} \lambda\left(S\left(f_{i}\right) ; R\right)^{k_{i}}}\right)=1-\frac{k_{1}}{\rho}+o(1) .
$$

Thus, we obtain

$$
\log _{R} F_{1}(R)-\log _{R} F_{2}(R) \leq G_{1+k_{1}}\left(\frac{1}{\rho}\right)+o(1)
$$

as $R$ tends to infinity. For the proof of (4.2), it suffices to show that

$$
G_{1+k_{1}}\left(\frac{1}{\rho}\right)<0
$$

In fact, (5.16) implies that there exists a positive constant $c$ satisfying

$$
F_{1}(R)<R^{-c} F_{2}(R)
$$

for any sufficiently large $R$.

If $k_{1}=0$ or $k_{1}=1$, then (5.16) is clear by $G_{1}(X)=-X$ and $G_{2}(X)=$ $-X(1-X)$. If $k_{1}=2$, then we have $G_{3}(X)=-X\left(1-3 X+X^{2}\right)$ and $\sigma_{3}=$ $(3-\sqrt{5}) / 2$. By (5.10) and (4.1), we get

$$
\frac{1}{\rho}<\frac{1}{A} \leq \frac{1}{1+k_{1}}=\frac{1}{3}<\sigma_{3},
$$

which implies (5.16) by the second statement of Lemma 3.6. Finally, suppose that $k_{1} \geq 3$. Using (3.2), (4.1), and the third statement of Lemma 3.6, we obtain

$$
\frac{1}{\rho}<\sigma_{A} \leq \sigma_{1+k_{1}}
$$

which means (5.16). Therefore, we proved Theorem 3.7

Proof of Corollary 3.8 . The first statement of Corollary 3.8 follows from Theorem 3.7 by

$$
\sum_{m=0}^{\infty} X^{\left\lfloor y^{m}\right\rfloor \in \mathcal{F}}
$$

The second statement of the corollary is similarly verified by $\xi(y, z ; X) \in \mathcal{F}$. In fact, the second assumption on $\mathcal{F}$ follows from the fact that, for any real number $M$,

$$
\lim _{R \rightarrow \infty} \frac{\varphi(y, z ; R)}{R^{M}}=\infty .
$$


Moreover, in the same way as the proof of (5.4), we can show that

$$
\lim _{R \rightarrow \infty} \frac{\varphi(y, z ; R+1)}{\varphi(y, z ; R)}=1 .
$$

Proof of Corollary 3.9. By Theorem 3.7 and the second statement of Lemma [3.6. it suffices to show that $(\varepsilon+1 / 2) A^{2}>\sigma_{A}^{-1}$, namely,

$$
0>G_{A}\left(\left(\frac{1}{2}+\varepsilon\right)^{-1} A^{-2}\right)
$$

for any sufficiently large $A$, depending only on $\varepsilon>0$. We now fix an arbitrary positive real number $\varepsilon$. In the proof of Corollary 3.9, the implied constant in the symbol $O$ does not depend on $A$, but on $\varepsilon$. Observe that

$$
\begin{aligned}
& \log \left(1-\left(\frac{1}{2}+\varepsilon\right)^{-1} A^{-2}\right)^{A} \\
& =A\left(-\left(\frac{1}{2}+\varepsilon\right)^{-1} A^{-2}+O\left(A^{-4}\right)\right) \\
& =-\left(\frac{1}{2}+\varepsilon\right)^{-1} A^{-1}+O\left(A^{-3}\right)
\end{aligned}
$$

and that

$$
\begin{aligned}
& \left(1-\left(\frac{1}{2}+\varepsilon\right)^{-1} A^{-2}\right)^{A} \\
& =\exp \left(-\left(\frac{1}{2}+\varepsilon\right)^{-1} A^{-1}+O\left(A^{-3}\right)\right) \\
& =1-\left(\frac{1}{2}+\varepsilon\right)^{-1} A^{-1}+\frac{1}{2}\left(\frac{1}{2}+\varepsilon\right)^{-2} A^{-2}+O\left(A^{-3}\right) .
\end{aligned}
$$

Thus, we get

$$
\begin{aligned}
& G_{A}\left(\left(\frac{1}{2}+\varepsilon\right)^{-1} A^{-2}\right) \\
& =\left(1-\left(\frac{1}{2}+\varepsilon\right)^{-1} A^{-2}\right)^{A} \\
& \quad-1+\left(\frac{1}{2}+\varepsilon\right)^{-1} A^{-1}-\left(\frac{1}{2}+\varepsilon\right)^{-1} A^{-2} \\
& =-\varepsilon\left(\frac{1}{2}+\varepsilon\right)^{-2} A^{-2}+O\left(A^{-3}\right)<0
\end{aligned}
$$

for any sufficiently large $A$, depending only on $\varepsilon$. 


\section{Proof of our criteria}

\subsection{Proof of Theorem 4.2}

We prove Theorem 4.2 by Theorem 4.1, showing that

$$
f_{1}(X):=1+\sum_{m=m_{0}}^{\infty} X^{\lfloor a(m)\rfloor}, f_{2}(X):=1+\sum_{m=m_{0}}^{\infty} X^{\lfloor u(m)\rfloor}
$$

satisfy the assumptions of Theorem 4.1, where $r=2$ and $A$ is any fixed positive integer. The first assumption is trivial. The fourth assumption of Theorem 4.1 follows from the first assumption on $u(R)$. Using (4.3) and the second assumption on $u(R)$, we get the following: For any positive real number $\varepsilon$,

$$
\begin{aligned}
& \lambda\left(S\left(f_{1}\right) ; R\right) \sim b(R)=o\left(R^{\varepsilon}\right), \\
& \lambda\left(S\left(f_{2}\right) ; R\right) \sim v(R)=o\left(b(R)^{\varepsilon}\right)=o\left(\lambda\left(S\left(f_{1}\right) ; R\right)^{\varepsilon}\right)
\end{aligned}
$$

as $R$ tends to infinity, which implies that the third assumption on Theorem 4.1 holds.

In what follows, we check the second assumption. In the same way as the proof of Lemma 5.2, we show the following:

LEMMA 6.1. Let $k$ be a positive integer and $\varepsilon$ a positive real number. Then we have

$$
R-\theta\left(R ; k S\left(f_{1}\right)\right) \ll \frac{R}{b(R)^{k-\varepsilon}}
$$

for any $R \geq 1$, where the implied constant in the symbol $\ll$ does not depend on $R$, but on $k$ and $\varepsilon$.

Proof. It suffices to show for each $k \geq 1$ that, for any $\varepsilon>0$, 6.3 holds for any sufficiently large $R$, depending on $k$ and $\varepsilon$. We prove the lemma by induction on $k$.

We first consider the case of $k=1$. We may assume that $\varepsilon<1$. By the second assumption on $a(m)$ and the mean value theorem, we get for any sufficiently large $m$ that

$$
a(m) \leq a(m+1) \leq 2 a(m)
$$

and that there exists a real number $\rho$ with $0<\rho<1$ satisfying

$$
\begin{aligned}
a(m+1)-a(m) & =a^{\prime}(m+\rho)<\frac{a(m+\rho)}{(m+\rho)^{1-\varepsilon}} \\
& \leq \frac{a(m+1)}{m^{1-\varepsilon}} \ll \frac{a(m)}{(m+1)^{1-\varepsilon}} .
\end{aligned}
$$

For any sufficiently large $R$, there exists an integer $m \geq m_{0}$ such that

$$
\lfloor a(m)\rfloor<R \leq\lfloor a(m+1)\rfloor .
$$

By (6.4), we obtain

$$
\begin{aligned}
R-\theta\left(R ; S\left(f_{1}\right)\right) & =R-\lfloor a(m)\rfloor \leq a(m+1)-a(m)+1 \\
& \ll \frac{a(m)}{(m+1)^{1-\varepsilon}} \ll \frac{R}{b(a(m+1))^{1-\varepsilon}} \leq \frac{R}{b(R)^{1-\varepsilon}},
\end{aligned}
$$


which implies (6.3) in the case of $k=1$.

Next we assume that (6.3) holds for a fixed positive integer $k$ and an arbitrary positive real number $\varepsilon$. In what follows, we verify (6.3) for $k+1$ with fixed $\varepsilon<1$. Put

$$
R_{0}:=R-\theta\left(R ; k S\left(f_{1}\right)\right) .
$$

It suffices to consider the case of

$$
R_{0} \geq \frac{R}{b(R)^{k+1}} .
$$

In fact, suppose that (6.5) does not hold. Since $0 \in S\left(f_{1}\right)$ by the definition of $f_{1}(X)$, we have

$$
\theta\left(R ; k S\left(f_{1}\right)\right) \in k S\left(f_{1}\right) \subset(k+1) S\left(f_{1}\right)
$$

by Remark 1 . Thus, we get

$$
R-\theta\left(R ;(k+1) S\left(f_{1}\right)\right) \leq R_{0}<\frac{R}{b(R)^{k+1}},
$$

which implies (6.3).

In what follows, we assume that (6.5) is satisfied. In particular, applying (6.1) to (6.5), we see

$$
R_{0} \geq R^{1-\varepsilon / 4}
$$

for any sufficiently large $R$. Moreover, the inductive hypothesis implies that

$$
R_{0} \ll \frac{R}{b(R)^{k-\varepsilon / 2}} .
$$

In the same way as the proof of Lemma 5.2 putting

$$
\eta:=\theta\left(R ; k S\left(f_{1}\right)\right)+\theta\left(R_{0} ; S\left(f_{1}\right)\right) \in(k+1) S\left(f_{1}\right),
$$

we see that

$$
\begin{aligned}
R-\theta\left(R ;(k+1) S\left(f_{1}\right)\right) & \leq R-\eta \\
& =R_{0}-\theta\left(R_{0} ; S\left(f_{1}\right)\right) \ll \frac{R_{0}}{b\left(R_{0}\right)^{1-\varepsilon / 4}},
\end{aligned}
$$

where for the last inequality we apply (6.3) with $k=1$. By (6.6) and (6.7), we obtain

$$
R-\theta\left(R ;(k+1) S\left(f_{1}\right)\right) \ll \frac{R}{b(R)^{k-\varepsilon / 2} b\left(R^{1-\varepsilon / 4}\right)^{1-\varepsilon / 4}} .
$$

Using the assumption that $(\log a(x)) /(\log x)$ is ultimately increasing with

$$
x=b(R)>x^{\prime}=b\left(R^{1-\varepsilon / 4}\right),
$$

we get

$$
\begin{aligned}
\frac{\log R}{\log b(R)} & =\frac{\log a(x)}{\log x} \geq \frac{\log a\left(x^{\prime}\right)}{\log x^{\prime}} \\
& =\left(1-\frac{\varepsilon}{4}\right) \frac{\log R}{\log b\left(R^{1-\varepsilon / 4}\right)}
\end{aligned}
$$


Consequently,

$$
b\left(R^{1-\varepsilon / 4}\right) \geq b(R)^{1-\varepsilon / 4},
$$

and so

$$
\frac{1}{b\left(R^{1-\varepsilon / 4}\right)^{1-\varepsilon / 4}} \leq \frac{1}{b(R)^{(1-\varepsilon / 4)^{2}}} \leq \frac{1}{b(R)^{1-\varepsilon / 2}}
$$

by $(1-\varepsilon / 4)^{2} \geq 1-\varepsilon / 2$. Combining (6.8) and (6.9), we deduce that

$$
(0<) R-\theta\left(R ;(k+1) S\left(f_{1}\right)\right) \ll \frac{R}{b(R)^{k+1-\varepsilon}},
$$

which implies (6.3).

Let $k_{1}, k_{2}$ be nonnegative integers. Applying Lemma 6.1] with $k=1+k_{1}$ and $\varepsilon=1 / 2$, we deduce by (6.2) that

$$
\begin{aligned}
R-\theta\left(R ;\left(1+k_{1}\right) S\left(f_{1}\right)\right) & \ll \frac{R}{b(R)^{k_{1}+1 / 2}} \\
& =o\left(\frac{R}{b(R)^{k_{1}} v(R)^{k_{2}}}\right)=o\left(\frac{R}{\prod_{h=1}^{2} \lambda\left(S\left(f_{h}\right) ; R\right)^{k_{h}}}\right)
\end{aligned}
$$

as $R$ tends to infinity. Finally, we proved Theorem 4.2 .

\subsection{Proof of Theorem 4.1}

Put

$$
\overline{f_{i}}(X):=\left\{\begin{array}{cc}
f_{i}(X) & \text { if } f_{i}(0) \neq 0 \\
1+f_{i}(X) & \text { if } f_{i}(0)=0
\end{array}\right.
$$

Then $\overline{f_{1}}(X), \ldots, \overline{f_{r}}(X)$ satisfy the assumptions of Theorem 4.1, The first and fourth assumptions are easily checked. Moreover, the second and the third assumptions are also seen by

$$
\begin{aligned}
& \theta\left(R ; \sum_{h=1}^{r-2} k_{h} S\left(\overline{f_{h}}\right)+\left(1+k_{r-1}\right) S\left(\overline{f_{r-1}}\right)\right) \\
& \quad \geq \theta\left(R ; \sum_{h=1}^{r-2} k_{h} S\left(f_{h}\right)+\left(1+k_{r-1}\right) S\left(f_{r-1}\right)\right)
\end{aligned}
$$

and, for $h=1, \ldots, r$,

$$
\lambda\left(S\left(\overline{f_{h}}\right) ; R\right) \sim \lambda\left(S\left(f_{h}\right) ; R\right)
$$

as $R$ tends to infinity. For the proof of Theorem 4.1, it suffices to show that

$$
\left\{\overline{f_{1}}\left(\beta^{-1}\right)^{k_{1}} \overline{f_{2}}\left(\beta^{-1}\right)^{k_{2}} \ldots \overline{f_{r}}\left(\beta^{-1}\right)^{k_{r}} \mid k_{1}, k_{2}, \ldots, k_{r} \in \mathbb{N}, k_{1} \leq A\right\}
$$

is linearly independent over $\mathbb{Q}(\beta)$. In particular, rewriting $\overline{f_{i}}(X)$ by $f_{i}(X)$ for $i=1, \ldots, r$, we may assume that $f_{i}(0) \neq 0$ for any $i=1, \ldots, r$. 
For simplicity, put, for $i=1, \ldots, r$,

$$
\xi_{i}:=f_{i}\left(\beta^{-1}\right), S_{i}:=S\left(f_{i}\right), \lambda_{i}(R):=\lambda\left(S\left(f_{i}\right) ; R\right) .
$$

Using Corollary 2.2 and the third assumption of Theorem 4.2 we see that

$$
\left[\mathbb{Q}\left(\xi_{1}, \beta\right): \mathbb{Q}(\beta)\right] \geq A+1
$$

and that $\xi_{2}, \ldots, \xi_{r}$ are transcendental.

We introduce notation for the proof of Theorem 4.1 For any nonempty subset $\mathcal{A}$ of $\mathbb{N}$ and any positive integer $k$, let $\mathcal{A}^{k}$ denote the $n$-fold Cartesian product. For convenience, set

$$
\mathcal{A}^{0}:=\{0\} .
$$

Let $k \in \mathbb{N}$ and $\boldsymbol{p}=\left(p_{1}, \ldots, p_{k}\right) \in \mathbb{N}^{k}$. We put

$$
|\boldsymbol{p}|:=\left\{\begin{array}{cc}
0 & (k=0), \\
p_{1}+\cdots+p_{k} & (k \geq 1)
\end{array}\right.
$$

and, for $i=1, \ldots, r$,

$$
t_{i}(\boldsymbol{p}):=\left\{\begin{array}{cc}
1 & (k=0), \\
t_{i}\left(p_{1}\right) \cdots t_{i}\left(p_{k}\right) & (k \geq 1) .
\end{array}\right.
$$

Moreover, for any $\boldsymbol{k}=\left(k_{1}, \ldots, k_{r}\right) \in \mathbb{N}^{r}$, let

$$
\underline{X}^{\boldsymbol{k}}=\prod_{i=1}^{r} X_{i}^{k_{i}}, \underline{\xi}^{\boldsymbol{k}}:=\prod_{i=1}^{r} \xi_{i}^{k_{i}}, \underline{\lambda}(N)^{\boldsymbol{k}}:=\prod_{i=1}^{r} \lambda_{i}(N)^{k_{i}} .
$$

We calculate $\xi^{k}$ in the same way as the proof of Theorem 2.1 in [12]. The method was inspired by the proof of Theorem 7.1 in $[3]$. Let $\boldsymbol{k} \in \mathbb{N}^{r} \backslash\{(0, \ldots, 0)\}$. Then we have

$$
\begin{aligned}
\underline{\xi}^{\boldsymbol{k}} & =\prod_{i=1}^{r}\left(\sum_{m_{i} \in S_{i}} t_{i}\left(m_{i}\right) \beta^{-m_{i}}\right)^{k_{i}} \\
& =\prod_{i=1}^{r} \sum_{\boldsymbol{m}_{i} \in S_{i}^{k_{i}}} t_{i}\left(\boldsymbol{m}_{i}\right) \beta^{-\left|\boldsymbol{m}_{i}\right|}=: \sum_{m=0}^{\infty} \beta^{-m} \rho(\boldsymbol{k} ; m),
\end{aligned}
$$

where

$$
\rho(\boldsymbol{k} ; m)=\sum_{\substack{m_{1} \in S_{1}^{k_{1}}, \ldots, m_{r} \in S_{r}^{k_{r}} \\\left|\boldsymbol{m}_{1}\right|+\cdots+\left|m_{r}\right|=m}} t_{1}\left(\boldsymbol{m}_{1}\right) \cdots t_{r}\left(\boldsymbol{m}_{r}\right) \in \mathbb{N} .
$$

Note that $\rho(\boldsymbol{k} ; m)$ is positive if and only if

$$
m \in \sum_{h=1}^{r} k_{h} S_{h} .
$$

We see that

$$
\rho(\boldsymbol{k} ; m) \leq \sum_{\substack{\boldsymbol{m}_{1} \in S_{1}^{k_{1}}, \ldots, \boldsymbol{m}_{r} \in S_{r}^{k_{r}} \\\left|\boldsymbol{m}_{1}\right|+\cdots+\left|\boldsymbol{m}_{r}\right|=m}} C_{7}^{|\boldsymbol{k}|} \leq C_{7}^{|\boldsymbol{k}|}(1+m)^{|\boldsymbol{k}|} .
$$

We give an analogue of Lemma 4.1 in 12 . 
LEMMA 6.2. Let $\boldsymbol{k} \in \mathbb{N}^{r} \backslash\{(0, \ldots, 0)\}$ and let $N \in \mathbb{Z}^{+}$. Then we have

$$
\sum_{m=0}^{N-1} \rho(\boldsymbol{k} ; m) \leq C_{7}^{|\boldsymbol{k}|} \underline{\lambda}(N)^{\boldsymbol{k}}
$$

and

$$
\operatorname{Card}\{m \in \mathbb{N} \mid m<N, \rho(\boldsymbol{k} ; m)>0\} \leq C_{7}^{|\boldsymbol{k}|} \underline{\lambda}(N)^{\boldsymbol{k}} .
$$

Proof. We see that (6.13) follows from (6.12) because $\rho(\boldsymbol{k} ; m) \in \mathbb{N}$ for any $m$. Put $S(i ; N):=S_{i} \cap[0, N)$ for $i=1, \ldots, r$. Then we get

$$
\begin{aligned}
\sum_{m=0}^{N-1} \rho(\boldsymbol{k} ; m) & =\sum_{\substack{\boldsymbol{m}_{1} \in S_{1}^{k_{1}}, \ldots, \boldsymbol{m}_{r} \in S_{r}^{k} \\
\left|\boldsymbol{m}_{1}\right|+\cdots+\left|\boldsymbol{m}_{r}\right|<N}} t_{1}\left(\boldsymbol{m}_{1}\right) \cdots t_{r}\left(\boldsymbol{m}_{r}\right) \\
& \leq C_{7}^{|\boldsymbol{k}|} \sum_{\substack{\boldsymbol{m}_{1} \in S(1 ; N)^{k_{1}} \\
\boldsymbol{m}_{2} \in S(2 ; N)^{k_{2}}}} \sum_{\boldsymbol{m}_{r} \in S(r ; N)^{k_{r}}} 1 \\
& =C_{7}^{|\boldsymbol{k}|} \underline{\lambda}(N)^{\boldsymbol{k}},
\end{aligned}
$$

which implies (6.12).

Assume that the set $\left\{\underline{\xi}^{\boldsymbol{k}} \mid \boldsymbol{k}=\left(k_{1}, \ldots, k_{r}\right) \in \mathbb{N}^{r}, k_{1} \leq A\right\}$ is linearly independent over $\mathbb{Q}(\beta)$. Then there exists $P\left(X_{1}, \ldots, X_{r}\right) \in \mathbb{Z}[\beta]\left[X_{1}, \ldots, X_{r}\right] \backslash \mathbb{Z}[\beta]$ such that the degree of $P\left(X_{1}, \ldots, X_{r}\right)$ in $X_{1}$ is at most $A$ and that

$$
P\left(\xi_{1}, \ldots, \xi_{r}\right)=0 .
$$

Let $D$ be the total degree of $P\left(X_{1}, \ldots, X_{r}\right)$. Without loss of generality, we may assume that $X_{r}\left(-1+X_{r}\right)$ divides $P\left(X_{1}, \ldots, X_{r}\right)$ and that if $r \geq 3$, then $X_{r-1}$ divides $P\left(X_{1}, \ldots, X_{r}\right)$. Put

$$
P\left(X_{1}, \ldots, X_{r}\right)=: \sum_{k \in \Lambda} A_{k} \underline{X}^{k}
$$

where $\Lambda$ is a nonempty finite subset of $\mathbb{N}^{r}$ and $A_{\boldsymbol{k}} \in \mathbb{Z}[\beta] \backslash\{0\}$ for any $\boldsymbol{k} \in \Lambda$. For any $\boldsymbol{k}=\left(k_{1}, \ldots, k_{r}\right) \in \Lambda$, we have $k_{r} \geq 1$ because $X_{r}$ divides $P\left(X_{1}, \ldots, X_{r}\right)$. Moreover, if $r \geq 3$, then

$$
k_{r-1} \geq 1
$$

because $X_{r-1}$ divides $P\left(X_{1}, \ldots, X_{r}\right)$.

The lexicographic order $\succ$ on $\mathbb{N}^{r}$ is defined as follows: Let $\boldsymbol{k}=\left(k_{1}, \ldots, k_{r}\right)$ and $\boldsymbol{k}^{\prime}=\left(k_{1}^{\prime}, \ldots, k_{r}^{\prime}\right)$ be distinct elements of $\mathbb{N}^{r}$. Put $l:=\min \left\{i \mid 1 \leq i \leq r, k_{i} \neq\right.$ $k_{i}^{\prime}$. Then $\boldsymbol{k} \succ \boldsymbol{k}^{\prime}$ if and only if $k_{l}>k_{l}^{\prime}$. The third assumption of Theorem 4.1 implies that if $\boldsymbol{k} \succ \boldsymbol{k}^{\prime}$, then

$$
\underline{\lambda}(N)^{\boldsymbol{k}^{\prime}}=o\left(\underline{\lambda}(N)^{\boldsymbol{k}}\right)
$$

as $N$ tends to infinity. 
Let $\boldsymbol{g}=\left(g_{1}, \ldots, g_{r}\right)$ be the greatest element of $\Lambda$ with respect to $\succ$. Without loss of generality, we may assume that

$$
A_{g} \geq 1
$$

We see that

$$
g_{r-1} \geq 1 .
$$

In fact, (6.19) follows from (6.16) if $r \geq 3$. Suppose that $r=2$. Then $g_{1}$ is the degree of $P\left(X_{1}, X_{2}\right)$ in $X_{1}$. Thus, $g_{1}$ is positive because $\xi_{2}$ is transcendental.

Putting

$$
\Lambda_{1}:=\left\{\boldsymbol{k}=\left(k_{1}, \ldots, k_{r-1}, k_{r}\right) \mid k_{1}=g_{1}, \ldots, k_{r-1}=g_{r-1}, k_{r}<g_{r}\right\}
$$

and

$$
\Lambda_{2}:=\left\{\boldsymbol{k}=\left(k_{1}, \ldots, k_{r-1}, k_{r}\right) \mid k_{i}<g_{i} \text { for some } i \leq r-1\right\},
$$

we see $\Lambda=\{\boldsymbol{g}\} \cup \Lambda_{1} \cup \Lambda_{2}$. Using the fact that $\xi_{r}$ is transcendental and that $-1+X_{r}$ divides $P\left(X_{1}, \ldots, X_{r}\right)$, we obtain the following lemma, applying the same method as the proof of Lemma 4.3 in [12] with $F\left(X_{r-1}, X_{r}\right)=1$ :

LEMMA 6.3. $\Lambda_{1}$ and $\Lambda_{2}$ are not empty.

Set

$$
\boldsymbol{e}=\left(g_{1}, \ldots, g_{r-2},-1+g_{r-1}, 1+D\right) .
$$

Recall that the degree $g_{1}$ of $P\left(X_{1}, \ldots, X_{r}\right)$ in $X_{1}$ is at most $A$. Thus, we can apply the second assumption of Theorem 4.1 with $\boldsymbol{k}=\left(k_{1}, \ldots, k_{r}\right)=\boldsymbol{e}$. In fact, we see

$$
k_{1}=\left\{\begin{array}{cc}
-1+g_{1} & (\text { if } r=2), \\
g_{1} & (\text { if } r \geq 3) .
\end{array}\right.
$$

Hence, there exits a positive constant $C_{12}$ satisfying the following: For any integer $R$ with $R \geq C_{12}$, we have

$$
\lambda_{r}(R) \geq 5
$$

and

$$
R-\theta\left(\sum_{h=1}^{r-1} g_{h} S_{h} ; R\right)<\frac{R}{\underline{\lambda}(R)^{e}} .
$$

In what follows, we set

$$
\theta(R):=\theta\left(\sum_{h=1}^{r-1} g_{h} S_{h} ; R\right)
$$

for simplicity. Using (6.20) and (6.21), we obtain the following lemma in the same way as the proof of Lemma 4.4 in [12]: 
LEMMA 6.4. Let $M, E$ be real numbers with

$$
M \geq C_{12}, E \geq \frac{4 M}{\underline{\lambda}(M)^{e}} .
$$

Then

$$
M+\frac{1}{2} E<\theta(M+E)
$$

Using $k_{1} \leq A$ and the third assumption of Theorem 4.1, we get

$$
\lim _{R \rightarrow \infty} \frac{R}{\underline{\lambda}(R)^{e}}=\infty .
$$

Thus, the set

$$
\Xi:=\left\{N \in \mathbb{N} \mid \frac{N}{\underline{\lambda}(N)^{e}} \geq \frac{n}{\underline{\lambda}(n)^{e}} \text { for any } n \leq N\right\}
$$

is infinite. We now verify for any $\boldsymbol{k}=\left(k_{1}, \ldots, k_{r}\right) \in \Lambda_{2}$ that

$$
\underline{\lambda}(N)^{\boldsymbol{k}}=o\left(\underline{\lambda}(N)^{\boldsymbol{e}}\right)
$$

as $N$ tends to infinity. For the proof of (6.22), it suffices to check

$$
e \succ k
$$

by (6.17). If $g_{i}>k_{i}$ for some $i \leq r-2$, then (6.23) holds. Suppose that $g_{i}=k_{i}$ for any $i \leq r-2$. Then we get $-1+g_{r-1} \geq k_{r-1}$ and $1+D>k_{r}$ by $\boldsymbol{k} \in \Lambda_{2}$, which implies (6.23).

Combining (6.14), (6.15), and (6.10), we get

$$
0=\sum_{k \in \Lambda} A_{\boldsymbol{k}} \underline{\xi}^{\boldsymbol{k}}=\sum_{\boldsymbol{k} \in \Lambda} A_{\boldsymbol{k}} \sum_{m=0}^{\infty} \rho(\boldsymbol{k} ; m) \beta^{-m} .
$$

For an arbitrary nonnegative integer $R$, multiplying $\beta^{R}$ to the both-hand sides of the equality above, we obtain

$$
0=\sum_{\boldsymbol{k} \in \Lambda} A_{\boldsymbol{k}} \sum_{m=-R}^{\infty} \rho(\boldsymbol{k} ; m+R) \beta^{-m} .
$$

Putting

$$
\begin{aligned}
Y_{R} & :=\sum_{\boldsymbol{k} \in \Lambda} A_{\boldsymbol{k}} \sum_{m=1}^{\infty} \rho(\boldsymbol{k} ; m+R) \beta^{-m} \\
& =-\sum_{\boldsymbol{k} \in \Lambda} A_{\boldsymbol{k}} \sum_{m=-R}^{0} \rho(\boldsymbol{k} ; m+R) \beta^{-m},
\end{aligned}
$$

we see that $Y_{R}$ is an algebraic integer because $\beta$ is a Pisot or Salem number. 
LEMMA 6.5. There exist positive integers $C_{13}$ and $C_{14}$ satisfying the following: For any integer $R$ with $R \geq C_{14}$, we have

$$
Y_{R}=0 \text {, or }\left|Y_{R}\right| \geq R^{-C_{13}} .
$$

Proof. Let $d$ be the degree of $\beta$ and let $\sigma_{1}, \sigma_{2}, \ldots, \sigma_{d}$ be the conjugate embeddings of $\mathbb{Q}(\beta)$ into $\mathbb{C}$ such that $\sigma_{1}(\gamma)=\gamma$ for any $\gamma \in \mathbb{Q}(\beta)$. Set

$$
C_{15}:=\max \left\{\left|\sigma_{i}\left(A_{\boldsymbol{k}}\right)\right| \mid i=1, \ldots, d, \boldsymbol{k} \in \Lambda\right\} .
$$

Let $2 \leq i \leq d$. Using (6.24) and (6.11), and $\left|\beta_{i}\right| \leq 1$, we get

$$
\begin{aligned}
\left|\sigma\left(Y_{R}\right)\right| & =\left|\sum_{\boldsymbol{k} \in \Lambda} \sigma_{i}\left(A_{\boldsymbol{k}}\right) \sum_{n=0}^{R} \rho(\boldsymbol{k} ;-n+R) \sigma_{i}(\beta)^{n}\right| \\
& \leq \sum_{\boldsymbol{k} \in \Lambda} C_{15} \sum_{n=0}^{R} C_{7}^{D}(1+R)^{D} \ll(R+1)^{D+1} .
\end{aligned}
$$

In particular, if $R \gg 1$, then

$$
\left|\sigma\left(Y_{R}\right)\right| \leq R^{D+2} .
$$

Hence, if $Y_{R} \neq 0$, then we obtain

$$
1 \leq\left|Y_{R}\right| \prod_{i=2}^{d}\left|\sigma\left(Y_{R}\right)\right| \leq\left|Y_{R}\right| R^{(D+2)(d-1)} .
$$

In the case of $\beta=2$ and $r=1$, Bailey, Borwein, Crandall, and Pomerance estimated the numbers $\widetilde{y_{N}}$ of positive $Y_{R}$ with $R<N$ in order to give lower bounds for the nonzero digits in binary expansions (Theorem 7.1 in 3 ). Moreover, if $\beta=b>1$ is a rational integer and $r \geq 2$, then $\widetilde{y_{N}}$ is applied to prove a criterion for algebraic independence (Theorem 2.1 in [12]).

Now, we put, for $N \in \mathbb{Z}^{+}$,

$$
y_{N}:=\operatorname{Card}\left\{\begin{array}{l|l}
R \in \mathbb{N} & \left.R<N, Y_{R} \geq \frac{1}{\beta}\right\} .
\end{array}\right.
$$

In the case where $\beta$ is a Pisot or Salem number and $r=1$, then $y_{N}$ is estimated to give lower bounds for the numbers of nonzero digits in $\beta$-expansions (Theorem 2.2 in [14). In what follows, we calculate upper and lower bounds for $y_{N}$, which gives contradiction. First, we estimate upper bounds for $y_{N}$ in Lemma 6.6, Next, we give lower bounds for $y_{N}$ in Lemma 6.11] estimating upper bounds for $R-\theta(R ; \Omega)$ in Lemma 6.10, where

$$
\Omega=\left\{R \in \mathbb{N} \mid Y_{R} \geq \frac{1}{\beta}\right\} .
$$

In what follows, we assume that $N$ is a sufficiently large integer satisfying

$$
\left(1+\frac{1}{N}\right)^{D}<\frac{\beta+1}{2} \text {. }
$$


LEMMA 6.6. We have

$$
y_{N}=o\left(N^{1-\delta / 2}\right)
$$

as $N$ tends to infinity.

Proof. Put

$$
K:=\left\lceil(1+D) \log _{\beta} N\right\rceil .
$$

Then we see

$$
\begin{aligned}
y_{N} & \leq K+y_{N-K}=K+\sum_{\substack{0 \leq R<N-K \\
Y_{R} \geq 1 / \beta}} 1 \\
& \leq K+\beta \sum_{R=0}^{N-K-1}\left|Y_{R}\right|
\end{aligned}
$$

and

$$
\begin{aligned}
\sum_{R=0}^{N-K-1}\left|Y_{R}\right| & \leq \sum_{R=0}^{N-K-1} \sum_{\boldsymbol{k} \in \Lambda} \sum_{m=1}^{\infty}\left|A_{\boldsymbol{k}}\right| \beta^{-m} \rho(\boldsymbol{k} ; m+R) \\
& =\sum_{\boldsymbol{k} \in \Lambda}\left|A_{\boldsymbol{k}}\right| Y(\boldsymbol{k} ; N),
\end{aligned}
$$

where

$$
Y(\boldsymbol{k} ; N)=\sum_{R=0}^{N-K-1} \sum_{m=1}^{\infty} \beta^{-m} \rho(\boldsymbol{k} ; m+R)
$$

for $\boldsymbol{k} \in \Lambda$. For the proof of Lemma 6.6. it suffices to show for any $\boldsymbol{k}=$ $\left(k_{1}, k_{2}, \ldots, k_{r}\right) \in \Lambda$ that

$$
Y(\boldsymbol{k} ; N)=o\left(N^{1-\delta / 2}\right)
$$

as $N$ tends to infinity. Observe that

$$
\begin{aligned}
0 \leq Y(\boldsymbol{k} ; N) & =\sum_{m=1}^{K} \sum_{R=0}^{N-K-1} \beta^{-m} \rho(\boldsymbol{k} ; m+R) \\
& \quad+\sum_{m=K+1}^{\infty} \sum_{R=0}^{N-K-1} \beta^{-m} \rho(\boldsymbol{k} ; m+R) \\
= & : S^{(1)}(\boldsymbol{k} ; N)+S^{(2)}(\boldsymbol{k} ; N) .
\end{aligned}
$$

Using (6.12), we get

$$
\begin{aligned}
S^{(1)}(\boldsymbol{k} ; N) & \leq \sum_{m=1}^{K} \beta^{-m} \sum_{R=0}^{N-1} \rho(\boldsymbol{k} ; R) \leq \sum_{m=1}^{\infty} \beta^{-m} \sum_{R=0}^{N-1} \rho(\boldsymbol{k} ; R) \\
& \leq \sum_{m=1}^{\infty} \beta^{-m} C_{7}^{D} \underline{\lambda}(N)^{\boldsymbol{k}} \ll \underline{\lambda}(N)^{\boldsymbol{k}} .
\end{aligned}
$$


Thus, the third assumption of Theorem 4.1 implies that

$$
S^{(1)}(\boldsymbol{k} ; N) \ll \lambda_{1}(N)^{A} \prod_{i=2}^{r} \lambda_{i}(N)^{k_{i}}=o\left(N^{1-\delta / 2}\right) .
$$

Using (6.11), we see

$$
\begin{aligned}
S^{(2)}(\boldsymbol{k} ; N) & \leq \sum_{m=K+1}^{\infty} \beta^{-m} \sum_{R=0}^{N-K-1} C_{7}^{D}(m+R+1)^{D} \\
& \ll \sum_{m=K+1}^{\infty} \beta^{-m} N(m+N)^{D} .
\end{aligned}
$$

Note for any $m \in \mathbb{N}$ that

$$
\left(\frac{m+1+N}{m+N}\right)^{D} \leq\left(1+\frac{1}{N}\right)^{D}<\frac{\beta+1}{2}
$$

by (6.26). Hence, we obtain

$$
\begin{aligned}
S^{(2)}(\boldsymbol{k} ; N) & \ll \beta^{-K-1} N(K+1+N)^{D} \sum_{m=0}^{\infty} \beta^{-m}\left(\frac{\beta+1}{2}\right)^{m} \\
& \ll \beta^{-K-1} N^{D+1} \leq 1 .
\end{aligned}
$$

Hence, combining (6.28), (6.29), and (6.30), we deduce (6.27).

In what follows, we estimate lower bounds for $y_{N}$ in the case where $N \in \Xi$ is sufficiently large. Recall that $\Lambda_{2}$ is not empty by Lemma 6.3 and that $0 \in S_{i}$ for $i=1, \ldots, r$. In particular, for any $\boldsymbol{k} \in \Lambda$, we have $\rho(\boldsymbol{k} ; 0)>0$. Put

$$
\begin{array}{r}
\left\{T \in \mathbb{N} \mid T<N, \rho(\boldsymbol{k} ; T)>0 \text { for some } \boldsymbol{k} \in \Lambda_{2}\right\} \\
=:\left\{0=T_{1}<T_{2}<\cdots<T_{\tau}\right\} .
\end{array}
$$

If $N$ is sufficiently large, then (6.13) and (6.22) imply that

$$
\tau \leq \sum_{\boldsymbol{k} \in \Lambda_{2}} C_{7}^{|\boldsymbol{k}|} \underline{\lambda}(N)^{\boldsymbol{k}} \leq \frac{1}{32} \underline{\lambda}(N)^{e} .
$$

For convenience, put $T_{1+\tau}:=N$. Set

$$
\mathcal{J}:=\{J=J(j) \mid 1 \leq j \leq \tau\},
$$

where $J(j)$ is an interval of $\mathbb{R}$ defined by $J(j)=\left[T_{j}, T_{1+j}\right)$ for $1 \leq j \leq \tau$.

In what follows, we denote the length of a bounded interval $I$ of $\mathbb{R}$ by $|I|$. Then we have

$$
\sum_{J \in \mathcal{J}}|J|=N
$$

Let

$$
\begin{aligned}
& \mathcal{J}_{1}:=\left\{J \in \mathcal{J}|| J \mid \geq \frac{16 N}{\underline{\lambda}(N)^{e}}\right\}, \\
& \mathcal{J}_{2}:=\left\{J \in \mathcal{J}_{1} \mid J \subset\left[C_{12}, N\right)\right\} .
\end{aligned}
$$

In the same way as the proof of Lemma 4.7 in [12, we obtain the following: 
LEMMA 6.7. If $N \in \Xi$ is sufficiently large, then we have

$$
\sum_{J \in \mathcal{J}_{1}}|J| \geq \frac{N}{2}, \quad \sum_{J \in \mathcal{J}_{2}}|J| \geq \frac{N}{3} .
$$

Recall that $\Lambda_{1}$ is not empty by Lemma 6.3. Let $\boldsymbol{k}_{1}$ be the maximal element of $\Lambda_{1}$ with respect to $\succ$. Set

$$
\begin{array}{r}
\left\{R \in \mathbb{N} \mid R<N, \rho(\boldsymbol{k} ; R)>0 \text { for some } \boldsymbol{k} \in \Lambda_{1}\right\} \\
=:\left\{0=R_{1}<R_{2}<\cdots<R_{\mu}\right\}
\end{array}
$$

and $R_{1+\mu}:=N$. Then (6.13) implies that

$$
\mu \leq \sum_{\boldsymbol{k} \in \Lambda_{1}} C_{7}^{|\boldsymbol{k}|} \underline{\lambda}(N)^{\boldsymbol{k}} \leq C_{16} \underline{\lambda}(N)^{\boldsymbol{k}_{1}},
$$

where $C_{16}$ is a positive constant.

Let

$$
\mathcal{I}:=\{I=I(i) \mid 1 \leq i \leq \mu\},
$$

where $I(i)$ is an interval of $\mathbb{R}$ defined by $I(i)=\left[R_{i}, R_{i+1}\right)$ for $1 \leq i \leq \mu$. Set

$$
y_{N}(i):=\operatorname{Card}\left\{R \in I(i) \mid Y_{R} \geq \frac{1}{\beta}\right\}
$$

for $i=1, \ldots, \mu$. Observe that

$$
\sum_{I \in \mathcal{I}}|I|=N
$$

and that

$$
\sum_{i=1}^{\mu} y_{N}(i)=y_{N}
$$

Set

$$
\begin{aligned}
& \mathcal{I}_{1}:=\{I \in \mathcal{I} \mid I \subset J \text { for some } J \in \mathcal{J}\}, \\
& \mathcal{I}_{2}:=\left\{I \in \mathcal{I}_{1}|| I \mid \geq \frac{1}{12 C_{16}} \frac{N}{\underline{\lambda}(N)^{\boldsymbol{k}_{1}}}\right\} .
\end{aligned}
$$

In the same way as the proof of Lemma 4.8 in [12, we obtain the following:

LEMMA 6.8. For any sufficiently large $N \in \Xi$, we have

$$
\sum_{I \in \mathcal{I}_{1}}|I| \geq \frac{N}{6}, \quad \sum_{I \in \mathcal{I}_{2}}|I| \geq \frac{N}{12} .
$$


In what follows, we assume that $N \in \Xi$ satisfies

$$
N^{\delta / 2} \geq\left(1+C_{8}\right) C_{9}
$$

Let $1 \leq i \leq \mu$ with $I(i) \in \mathcal{I}_{2}$ and let $R \in\left(R_{i}, R_{i+1}\right)$. We now show that

$$
\rho(\boldsymbol{k} ; R)=0
$$

for any $\boldsymbol{k} \in \Lambda_{1} \cup \Lambda_{2}=\Lambda \backslash\{\boldsymbol{g}\}$. In fact, if $\boldsymbol{k} \in \Lambda_{1}$, then (6.34) follows from the definition of $R_{1}, \ldots, R_{\mu+1}$. Suppose that $\boldsymbol{k} \in \Lambda_{2}$. By the definition of $\mathcal{I}_{2}$, we have $I(i) \subset J(j)$ for some $j$ with $1 \leq j \leq \tau$, and so $R \in\left(T_{j}, T_{1+j}\right)$. Thus, we get 6.34).

Applying the third assumption of Theorem 4.1 with $\varepsilon=\delta /(2 D)$, we see by $g_{1} \leq A$ that

$$
\underline{\lambda}(N)^{\boldsymbol{k}_{1}}=o\left(N^{-\delta / 2+1}\right)
$$

as $N \in \Xi$ tends to infinity. Thus, we obtain for any sufficiently large $N \in \Xi$ that

$$
|I(i)| \geq \frac{1}{12 C_{16}} \frac{N}{\underline{\lambda}(N)^{\boldsymbol{k}_{1}}} \geq N^{\delta / 2} .
$$

We can apply the fourth assumption of Theorem 4.1 with

$$
R=\frac{|I(i)|}{1+C_{8}} \geq \frac{N^{\delta / 2}}{1+C_{8}} \geq C_{9}
$$

by (6.35) and (6.33). Thus, we get that there exists $V(N, i) \in S_{r}$ with

$$
\frac{|I(i)|}{1+C_{8}} \leq V(N, i) \leq \frac{C_{8}|I(i)|}{1+C_{8}}
$$

Put $M=M(N, i):=R_{i}+V(N, i)$. Then we have

$$
R_{i}+\frac{|I(i)|}{1+C_{8}} \leq M \leq R_{i}+\frac{C_{8}|I(i)|}{1+C_{8}} .
$$

By the definition of $R_{i}$, there exists $k_{r} \leq-1+g_{r}$ such that

$$
R_{i} \in \sum_{h=1}^{r-1} g_{h} S_{h}+k_{r} S_{r} .
$$

Using Remark 1 we see

$$
R_{i} \in \sum_{h=1}^{r-1} g_{h} S_{h}+\left(-1+g_{r}\right) S_{r} .
$$

Thus, we get

$$
M \in \sum_{h=1}^{r} g_{h} S_{h}
$$

by $V(N, i) \in S_{r}$. 
LEMMA 6.9. Let $N \in \Xi$ be sufficiently large and let $1 \leq i \leq \mu$ with $I(i) \in \mathcal{I}_{2}$. Then $Y_{R}>0$ for any $R$ with $R_{i} \leq R<M$.

Proof. We prove Lemma 6.9 by induction on $R$. First we show that $Y_{M-1}>0$. We see

$$
\begin{aligned}
Y_{M-1}= & A_{\boldsymbol{g}} \sum_{m=1}^{\infty} \beta^{-m} \rho(\boldsymbol{g} ; m+M-1) \\
& +\sum_{\boldsymbol{k} \in \Lambda \backslash\{\boldsymbol{g}\}} A_{\boldsymbol{k}} \sum_{m=1}^{\infty} \beta^{-m} \rho(\boldsymbol{k} ; m+M-1) \\
=: & S^{(3)}+S^{(4)} .
\end{aligned}
$$

By (6.37)

$$
S^{(3)} \geq \frac{A_{\boldsymbol{g}}}{\beta} \rho(\boldsymbol{g} ; M) \geq \frac{1}{\beta} .
$$

We now estimate upper bounds for $\left|S_{4}\right|$. Let $m$ be an integer with

$$
1 \leq m \leq-1+\left\lceil 2 D \log _{\beta} N\right\rceil .
$$

Using (6.36) and (6.35), we get

$$
\begin{aligned}
R_{i+1}-M & \geq R_{i+1}-R_{i}-\frac{C_{8}|I(i)|}{1+C_{8}} \\
& =\frac{|I(i)|}{1+C_{8}}>m
\end{aligned}
$$

for sufficiently large $N \in \Xi$ and

$$
R_{i+1}>m+M-1>R_{i} .
$$

Thus, applying (6.34) with $R=m+M-1$ for any $m$ with (6.40), we obtain by (6.11) that

$$
\begin{aligned}
\left|S^{(4)}\right| & \leq \sum_{\boldsymbol{k} \in \Lambda \backslash\{\boldsymbol{g}\}}\left|A_{\boldsymbol{k}}\right| \sum_{m=\left\lceil 2 D \log _{\beta} N\right\rceil}^{\infty} \beta^{-m} \rho(\boldsymbol{k} ; m+M-1) \\
& \leq \sum_{\boldsymbol{k} \in \Lambda \backslash\{\boldsymbol{g}\}}\left|A_{\boldsymbol{k}}\right| \sum_{m=\left\lceil 2 D \log _{\beta} N\right\rceil}^{\infty} \beta^{-m} C_{7}^{D}(m+N)^{D} \\
& \ll \sum_{m=\left\lceil 2 D \log _{\beta} N\right\rceil}^{\infty} \beta^{-m}(m+N)^{D} .
\end{aligned}
$$

Therefore, (6.26) implies that

$$
\left|S^{(4)}\right| \ll N^{-2 D}\left(\left\lceil 2 D \log _{\beta} N\right\rceil+N\right)^{D} \sum_{m=0}^{\infty} \beta^{m}\left(\frac{1+\beta}{2}\right)^{m}=o(1)
$$

as $N$ tends to infinity. In particular, if $N \in \Xi$ is sufficiently large, then

$$
\left|S^{(4)}\right|<\frac{1}{2 \beta} \text {. }
$$


Combining (6.38), (6.39), and (6.41), we deduce that if $N \in \Xi$ is sufficiently large, then $Y_{M-1}>0$.

Next, we assume that $Y_{R}>0$ for some $R$ with $R_{i}<R<M$. Using (6.34), we see

$$
\begin{aligned}
Y_{R-1} & =\sum_{\boldsymbol{k} \in \Lambda} A_{\boldsymbol{k}} \frac{1}{\beta} \rho(\boldsymbol{k} ; R)+\sum_{\boldsymbol{k} \in \Lambda} A_{\boldsymbol{k}} \sum_{m=2}^{\infty} \beta^{-m} \rho(\boldsymbol{k} ; m+R-1) \\
& =\frac{A_{\boldsymbol{g}}}{\beta} \rho(\boldsymbol{g} ; R)+\frac{1}{\beta} \sum_{\boldsymbol{k} \in \Lambda} A_{\boldsymbol{k}} \sum_{m=1}^{\infty} \beta^{-m} \rho(\boldsymbol{k} ; m+R) \\
& =\frac{A_{\boldsymbol{g}}}{\beta} \rho(\boldsymbol{g} ; R)+\frac{1}{\beta} Y_{R} .
\end{aligned}
$$

By the inductive hypothesis

$$
Y_{R-1}>\frac{A_{\boldsymbol{g}}}{\beta} \rho(\boldsymbol{g} ; R) \geq 0 .
$$

Therefore, we proved Lemma 6.9

Recall that $\Omega$ is defined in (6.25).

LEMMA 6.10. Let $N \in \Xi$ be sufficiently large and let $1 \leq i \leq \mu$ with $I(i) \in \mathcal{I}_{2}$. Let $R$ be an integer with

$$
R_{i}+4 C_{13} \log _{\beta} N \leq R<M .
$$

Then we have

$$
R-\theta(R ; \Omega) \leq 2 C_{13} \log _{\beta} N .
$$

Proof. Put $R_{1}:=\theta(R ; \Omega)$. In the same way as the proof of (6.42), we see for any integer $n$ with $R_{i}<n<R_{i+1}$ that

$$
Y_{n-1}=\frac{A_{g}}{\beta} \rho(\boldsymbol{g} ; n)+\frac{1}{\beta} Y_{n} .
$$

First, we consider the case of $Y_{R} \geq 1$. Then (6.44) implies that

$$
Y_{R-1} \geq \frac{1}{\beta}
$$

and that $R-R_{1}=1$, which implies (6.43).

In what follows, we may assume that $0<Y_{R}<1$ by Lemma 6.9. Let $S:=\left\lceil C_{13} \log _{\beta} N\right\rceil$. Suppose for any integer $m$ with $0 \leq m \leq S$ that

$$
\rho(\boldsymbol{g} ; R-m)=0 .
$$

Noting $M>R>R-1>\cdots>R-S>R_{i}$, we get by (6.44) that

$$
1>Y_{R}=\beta Y_{R-1}=\cdots=\beta^{S} Y_{R-S}=\beta^{1+S} Y_{R-S-1}>0,
$$


where we use Lemma 6.9 for the last inequality by $R_{i}<R-S-1<M$. So we get

$$
\beta^{S+1}<Y_{R-S-1}^{-1}=\left|Y_{R-S-1}\right|^{-1} .
$$

Since

$$
R-S-1 \geq 2 C_{13} \log _{\beta} N>C_{14}
$$

for any sufficiently large $N$, we apply Lemma 6.5 as follows:

$$
\beta^{S+1}<\left|Y_{R-S-1}\right|^{-1} \leq(R-S-1)^{C_{13}}<N^{C_{13}} .
$$

Thus, we obtain

$$
\left\lceil C_{13} \log _{\beta} N\right\rceil+1=S+1<C_{13} \log _{\beta} N,
$$

a contradiction.

Hence, there exists an integer $m^{\prime}$ with $0 \leq m^{\prime} \leq S$ satisfying $\rho\left(\boldsymbol{g} ; R-m^{\prime}\right) \geq 1$. Applying (6.44) with $n=R-m^{\prime}$, we get by $Y_{R-m^{\prime}}>0$ that

$$
Y_{R-m^{\prime}-1} \geq \frac{A_{\boldsymbol{g}}}{\beta} \rho\left(\boldsymbol{g} ; R-m^{\prime}\right) \geq \frac{1}{\beta},
$$

where for the last inequality we use (6.18). Hence, we deduce that

$$
R-R_{1} \leq m^{\prime}+1 \leq 2 C_{13} \log _{\beta} N .
$$

LEMMA 6.11.

$$
\limsup _{N \rightarrow \infty} \frac{y_{N}}{\log N}>0 \text {. }
$$

Proof. Let $N \in \Xi$ be sufficiently large and let $1 \leq i \leq \mu$ with $I(i) \in \mathcal{I}_{2}$. Note that

$$
\lim _{N \rightarrow \infty} \frac{|I(i)|}{\log _{\beta} N}=\infty
$$

by (6.35). Combining (6.36), (6.45), and Lemma 6.10, we see that there exists a constant $C_{17}$ such that

$$
y_{N}(i) \geq C_{17} \frac{|I(i)|}{\log N} .
$$

Therefore, using (6.31) and (6.32), we obtain

$$
y_{N} \geq \sum_{\substack{1 \leq i \leq \mu \\ I(\bar{i}) \in \mathcal{I}_{2}}} y_{N}(i) \geq \sum_{I \in \mathcal{I}_{2}} C_{17} \frac{|I|}{\log N} \gg \frac{N}{\log N} .
$$

Finally, we deduce a contradiction from Lemma 6.6 and 6.11, which proves Theorem 4.1. 


\section{Acknowledgements}

This work was supported by JSPS KAKENHI Grant Number 15K17505.

\section{References}

[1] B. Adamczewski, Transcendance $\ll$ à la Liouville $\gg$ de certains nombres réels, C. R. Acad. Sci. Paris 338 (2004), 511-514.

[2] B. Adamczewski and C. Faverjon, Chiffres non nuls dans le développement en base entière des nombres algébriques irrationnels, C. R. Acad. Sci. Paris, 350 (2012), 1-4.

[3] D. H. Bailey, J. M. Borwein, R. E. Crandall and C. Pomerance, On the binary expansions of algebraic numbers, J. Théor. Nombres Bordeaux 16 (2004), 487-518.

[4] D. Bertrand. Theta functions and transcendence, The Ramanujan J. 1 (1997), 339-350.

[5] É. Borel, Sur les chiffres décimaux de $\sqrt{2}$ et divers problèmes de probabilités en chaîne, C. R. Acad. Sci. Paris 230 (1950), 591-593.

[6] Y. Bugeaud, Distribution modulo one and diophantine approximation, Cambridge Tracts in Math. 193, Cambridge, (2012).

[7] Y. Bugeaud, On the $b$-ary expansion of an algebraic number, Rend. Sem. Math. Univ. Padova 118 (2007), 217-233.

[8] P. Corvaja and U. Zannier, Some new applications of the subspace theorem, Compositio Math. 131 (2002), 319-340.

[9] S. Daniel, On gaps between numbers that are sums of three cubes, Mathematika 44 (1997), 1-13.

[10] A. Durand, Indépendance algébrique de nombres complexes et critère de transcendance, Compositio Math. 35 (1977), 259-267.

[11] D. Duverney, Ke. Nishioka, Ku. Nishioka, and I. Shiokawa. Transcendence of Jacobi's theta series, Proc. Japan. Acad. Sci, Ser. A bf 72 (1996), 202203.

[12] H. Kaneko, Algebraic independence of real numbers with low density of nonzero digits, Acta Arith 154 (2012), 325-351.

[13] H. Kaneko, On the beta-expansions of 1 and algebraic numbers for a Salem number beta, Ergod. Theory and Dynamical Syst. 35 (2015), 1243-1262.

[14] H. Kaneko, On the number of nonzero digits in the beta-expansions of algebraic numbers, to appear in Rend. Sem. Math. Univ. Padova.

[15] K. Nishioka, Algebraic independence by Mahler's method and $S$-unit equations, Compositio Math. 92 (1994), 87-110. 
[16] A. Rényi, Representations for real numbers and their ergodic properties, Acta Math. Acad. Sci. Hung. 8 (1957), 477-493.

[17] I. Shiokawa, Algebraic independence of certain gap series, Arch. Math. 38 (1982), 438-442.

[18] T. Tanaka, Algebraic independence of power series generated by linearly independent positive numbers, Results Math. 46(2004), 367-380.

Hajime Kaneko

Institute of Mathematics, University of Tsukuba, 1-1-1

Tennodai, Tsukuba, Ibaraki, 350-0006, JAPAN

Center for Integrated Research in Fundamental Science and Technology (CiRfSE)

University of Tsukuba, Tsukuba, Ibaraki, 305-8571, JAPAN

e-mail: kanekoha@math.tsukuba.ac.jp 